\title{
Optimized Assessment of Atrial Fibrillation Organization Through Suitable Parameters of Sample Entropy
}

\author{
Raúl Alcaraz*, Daniel Abásolo, Member, IEEE, Roberto Hornero, Member, IEEE and José J. Rieta, Member, IEEE
}

\begin{abstract}
Sample Entropy (SampEn) is a nonlinear regularity index that requires the $a$ priori selection of three parameters: the length of the sequences to be compared, $m$, the patterns similarity tolerance, $r$, and the number of samples under analysis, $N$. Appropriate values for $m, r$ and $N$ have been recommended in some cases, such as heart rate, hormonal data, etc., but no guidelines exist for the selection of that values. Hence, an optimal parameters study should be required for the application of SampEn to not previously analyzed biomedical signals. In this work, a thorough analysis on the optimal SampEn parameter values within two different scenarios of $\mathrm{AF}$ organization estimation, such as the prediction of paroxysmal AF termination and the electrical cardioversion outcome in persistent AF, is presented. Results indicated that, (i) the proportion between $N$ and the sampling rate $\left(f_{s}\right)$ should be higher than one second and $f_{s} \geq 256 \mathrm{~Hz}$, (ii) overlapping between adjacent $N$-length windows does not improve organization estimation and (iii) values of $m$ and $r$ maximizing classification should be considered within a range wider than the proposed in the literature for heart rate analysis, i. e. $m=1$ and $m=2$ and $r$ between 0.1 and 0.25 times the standard deviation of the data.
\end{abstract}

\section{INTRODUCTION}

$\mathbf{T}$ HE application of nonlinear regularity metrics to physiological signals is a valuable tool because "hidden information" related to underlying mechanisms can be obtained [1], [2]. To this respect, the employment of sample entropy (SampEn) to estimate non-invasively atrial fibrillation (AF) organization has revealed clinically useful information, which could be used for a better treatment of the arrhythmia [3]. The organization estimation of $\mathrm{AF}$ is a relevant aspect to improve its comprehension, since its mechanisms are still unexplained despite of affecting up to $1 \%$ of the general population [4].

Given a time series with $N$ data points, the a priori selection of two unknown parameters, $m$ and $r$, is required to compute SampEn [2]. The parameter $m$ determines the length of the sequences to be compared and $r$ is the tolerance for accepting similar patterns between two segments. Although these parameters are critical in determining the outcome of SampEn, no guidelines exist for optimizing their values. Typically recommended $m$ and $r$ values are $m=1$

\footnotetext{
${ }^{*} \mathrm{R}$. Alcaraz is with the Innovation in Bioengeeniering Research Group, University of Castilla-La Mancha, Campus Universitario, 16071, Cuenca, Spain (email: raul.alcaraz@uclm.es).

D. Abásolo and R. Hornero are with the Biomedical Engineering Group, University of Valladolid, Spain (email: danaba@yllera.tel.uva.es, robhor@tel.uva.es).

J. J. Rieta is with the Biomedical Synergy, Electronic Engineering Department, Universidad Politécnica de Valencia, Spain (email: jjrieta@ieee.org).
}

and $m=2$ and $r$ between 0.1 and 0.25 times the standard deviation (SD) of the data [5]. This recommendation is largely based on the application of approximate entropy (ApEn) to relatively slow dynamic signals such as heart rate [1], [5] and hormone secretion data [6]. Given that SampEn is a modified version of ApEn to solve its shortcomings, such as bias, relative inconsistency and dependence on the sample length [2], these values are also applicable to SampEn.

However, a recent work has demonstrated that the typically recommended values for ApEn are not always appropriate for fast dynamic signals [7]. As a consequence, since only few values, within the range suggested in the literature [5], were tested in the previous works where SampEn was applied to AF organization estimation [3], the main goal of the present study is to carry out an in depth analysis on SampEn parameters able to achieve optimized classification of AF events which are directly dependent on AF organization. Thus, several combinations of $m$ and $r$, over a range wider than the one typically recommended, together with the number of analyzed samples, $N$, and the overlapping effect between adjacent $N$-length windows have been analyzed. Additionally, since in AF organization estimation SampEn is applied to a time series that depends on the original ECG sampling rate $\left(f_{s}\right)$, the relationship between $N$ and $f_{s}$ is also addressed.

\section{Materials}

Two different scenarios, such as the prediction of paroxysmal AF termination the electrical cardioversion (ECV) outcome in persistent $\mathrm{AF}$, in which organization plays an important role, as corroborated by invasive recordings [8], were analyzed.

\section{A. Paroxysmal AF Database}

Fifty Holter recordings of 30 seconds in length and two leads (II and V1) available in Physionet [9] were analyzed. The database included non-terminating AF episodes (group $\mathrm{N}$ ), which were observed to continue in AF for, at least, one hour following the end of the excerpt, and AF episodes terminating immediately after the end of the extracted segment (group T). These signals were digitized at a sampling rate of $128 \mathrm{~Hz}$ and 16-bit resolution.

\section{B. Persistent AF Database}

Sixty-three patients with persistent AF lasting more than 30 days, undergoing ECV were followed during four weeks. 
A standard 12-lead ECG was acquired for each patient during the whole procedure and a segment of 30 seconds in length was extracted from each recording for the analysis. All the signals were digitized at a sampling rate of $1024 \mathrm{~Hz}$ and 16-bit resolution.

After ECV, 22 patients $(34.93 \%)$ maintained normal sinus rhythm (NSR) during the first month. In 31 patients (49.20\%), NSR duration was below one month and the remaining 10 (15.87\%), relapsed to AF immediately after ECV. These 41 patients constituted the group of AF recurrence. All the patients were under drug treatment with amiodarone.

\section{METHODS}

\section{A. AF organization estimation}

In both databases, lead $V_{1}$ was chosen for the analysis because previous works have shown that AA is prevalent in this lead [8]. To estimate AF organization, the application of SampEn to the surface ECG requires the fulfillment of several steps. Firstly, the ventricular activity has to be removed making use of a cancellation technique. Next, the main atrial wave (MAW) has to be extracted from the AA by applying a selective filtering centered on the dominant atrial frequency (DAF), i.e. the highest amplitude frequency within the 3-9 Hz range. Finally, SampEn computation can be applied to this wave. This approach has been described in detail in previous works [3].

\section{B. Selection of $N$ and $f_{s}$}

Previous works, in which heart rate and hormonal data regularity were analyzed [1], [5], [6], [10], have shown that for $m=2$, values of $r$ from 0.1 to 0.25 times the SD of the data and values of $N$ between 100 and 5000 samples produce good statistical validity of ApEn. Additionally, Pincus and Goldberger [1] suggested that $N$ should be at least $10^{\mathrm{m}}$ and, preferably, at least $30^{\mathrm{m}}$ when heart rate regularity is analyzed. However, this recommendation is not applicable to the case studied in this work, because the MAW length depends on the ECG sampling rate. To this respect, the selection of a specific number of samples $\mathrm{N}$ will stretch out more or less the MAW information as a function of the sampling rate. Hence, for high fs, a low MAW time interval would be analyzed and the opposite also holds.

Determining the optimal value of $N$ is relevant. When small $N$ values are used, the estimates can be inaccurate due to their large variance. On the contrary, large $N$ values may contain abrupt changes in amplitude that could result in inaccurate estimates due to normalization errors in the parameter $r$. However, it is noteworthy that $N$ depends on $f_{s}$, since $N=T \cdot f_{s}$, where $T$ denotes the time interval (in seconds) on which SampEn is computed. Thereby, in addition to the optimal $N$, it is important to determine the most appropriate sampling rate for the MAW.

To obtain the optimal values of these parameters, the MAW sampling rate was varied and, for each case, SampEn was computed with different $N$ values. More precisely, the selected $N$ values were $30,60,120,240,480,960,1920$, ..., $L$ samples, where $L$ is the closest series length lower than the analyzed MAW segment. Given that the minimum considered sampling rate was $64 \mathrm{~Hz}$ and that the ECG lengths were 30 seconds, their combination yields 1920 samples. As a consequence, all the selected $N$ values took that number as reference, divided or multiplied by a power of two. For each $N$, SampEn was computed with $m=2$ and $r=0.25$ times the SD of the analyzed segment. These values were chosen because they provided the best classification results in previous works when applying SampEn to AF [3].

The discriminative abilities for both predictions of paroxysmal AF termination and ECV result were calculated making use of the receiver operating characteristic (ROC) curves. The ROC curve is a graphical representation of the tradeoffs between sensitivity and specificity. The total number of paroxysmal AF patients and ECVs precisely classified was considered as the diagnostic accuracy corresponding to each prediction. As the number of episodes included into each database was not notably large, to evaluate the statistical robustness of the accuracy obtained for each prediction, a leave-one-out cross-validation scheme was used. Finally, statistically significant differences between groups were evaluated making use of Student's $t$-test.

The MAW was downsampled or upsampled to 64,128 , 256, 512, 1024 and $2048 \mathrm{~Hz}$. Since the original sampling rates were $128 \mathrm{~Hz}$ for paroxysmal $\mathrm{AF}$ and $1024 \mathrm{~Hz}$ for persistent AF, a method based on cubic splines was applied when interpolation was necessary. The effect of interpolation was tested by comparing SampEn between downsampled and interpolated ECV signals for each sampling rate. Results yielded a relative difference in SampEn between both sets lower than $0.085 \%$ in the worst case. As a consequence, it can be considered that the interpolation operation does not alter the result of SampEn.

\section{Selection of $m$ and $r$}

Once the optimal combinations of $N$ and $f_{s}$ were obtained, the most adequate selection of $m$ and $r$ was investigated. The accuracy and confidence of SampEn estimate improves as the number of length $m$ matches increases. The number of matches can be increased by choosing small $m$ (short templates) and large $r$ (wide tolerance). However, penalties appear when too relaxed criteria are used [10]. For small $r$ values, poor conditional probability estimates are achieved, while for large $r$ values, too much detailed system information is lost and SampEn tends to 0 for all the processes. To avoid a significant noise contribution on SampEn computation, one must choose $r$ larger than most of the noise [10]. Overall, to get optimal $m$ and $r$ values, an approach similar to the developed by Lake et al [11], [12] was used. SampEn was computed using a $10 \times 20$ matrix of combinations of $m=1,2, \ldots, 10$ and $r=0.05,0.1,0.15, \ldots, 1$ times the SD of the analyzed segment. Normalizing $r$ to the $\mathrm{SD}$ of the analyzed segment provides a translation and scale invariance, in the sense that SampEn remains unchanged under uniform process magnification, reduction, or constant shift to higher or lower values [5]. For each combination, sensitivity, specificity, accuracy and statistical significance were 
also computed for each prediction. Moreover, a leave-one-out cross-validation approach was applied to assess statistically the robustness of the diagnostic accuracy obtained for each combination of $m$ and $r$.

\section{RESUlts}

\section{A. Selection of $N$ and $f_{s}$}

Results showed that for a given $N$, when $f_{s}$ increases, SampEn decreases. On the other hand, for a given $f_{s}$ of $256 \mathrm{~Hz}$ and above, that SampEn increases when $N$ also increases up to a saturation point. Thus, for higher $N$, very similar SampEn values were obtained with variations lower than $\pm 5 \%$. On the contrary, for $f_{s}$ of $128 \mathrm{~Hz}$ and below, no clear increasing tendency was observed. In addition, the value of $N$ from which the limited increasing behavior was noticed was dependent on $f_{s}$. Thus, for rates of 256, 512, 1024 and $2048 \mathrm{~Hz}$, the reduced variation $N$ values for SampEn were 240, 480, 960 and 1920, respectively.

The discriminative differences between subsets of both databases were maintained for $f_{s}$ of $256 \mathrm{~Hz}$ and above. Indeed, a high predictive accuracy was achieved, such as Fig. 1 shows. Precisely, for $f_{s} \geq 256 \mathrm{~Hz}$ the lowest accuracy was $86 \%$ for paroxysmal $\mathrm{AF}$ and $73.02 \%$ for persistent AF, being $90 \%$ and $74.60 \%$ of cross-validated grouped cases correctly classified, respectively. In addition, differences between groups were noticed, given that a statistical significance lower than 0.01 was obtained for all the cases. On the contrary, for $f_{s} \leq 128 \mathrm{~Hz}$ a limited predictive ability was obtained for most pairs of $N$ and $f_{s}$. Moreover, in most of the cases, significant differences between groups were unnoticed because of their statistical significance $(p>0.01)$.

It is also noteworthy that for $N$ equal or higher than the saturation values for SampEn, the diagnostic accuracy presented a constant result of $92 \%$ for paroxysmal AF and $79.37 \%$ for persistent AF, which was higher than the obtained with lower $N$ values for a given $f_{s}$ (see Fig. 1). In addition, a higher statistical significance $(p<0.0001)$ together with an identification accuracy of $96 \%$ and $82.54 \%$ of cross-validated grouped cases, respectively, were also yielded.

\section{B. Selection of $m$ and $r$}

Results from the previous section showed that several combinations of $N$ and $f_{s}$ can provide good classification for both AF databases. Thereby, two different combinations were selected for the optimal selection of $m$ and $r$. In this case, the outcomes related to the diagnostic accuracy are presented in Figs. 2 and 3. A remarkable region in which a high accuracy for the two AF databases was reached can be appreciated. In addition, a very good statistical significance $(p<0.00001)$ was also obtained. As can be seen, the region was slightly larger for the pair $N=960$ samples and $f_{s}=1024 \mathrm{~Hz}$. This area can be approximately delimited, for both $\mathrm{AF}$ databases, by $m=1$ to $m=5$ and $r$ between 0.1 and 0.6 times the SD. Additionally, in all the cases, the optimal combination of $m$ and $r$ providing the highest diagnostic accuracy values was found within the indicated area.
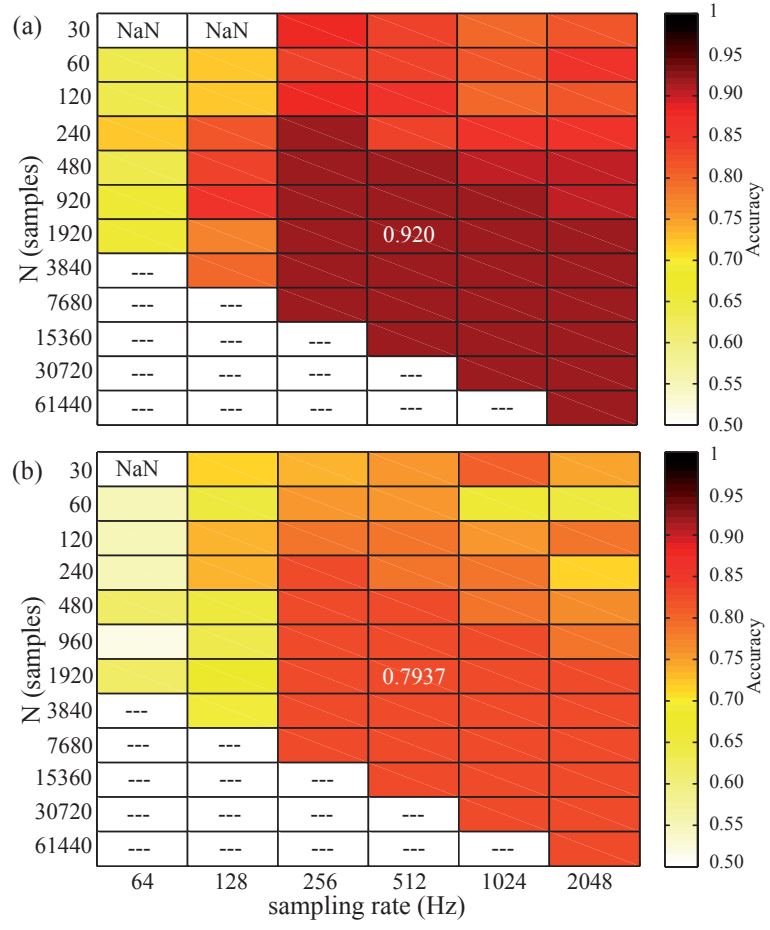

Fig. 1. Diagnostic accuracy color maps obtained with different combinations of $N$ and $f_{s}$ for (a) paroxysmal AF termination and (b) cardioversion of persistent AF. NaN in some cells stands for Not a Number because SampEn did not report similarity in such a short time series.

\section{DisCUSSION AND CONCLUSIONS}

Results showed that, for $f_{s}$ of $256 \mathrm{~Hz}$ and above, the differences between each subset, both for paroxysmal and persistent AF, were maintained independently of $N$. On the other hand, for a specific $f_{s}$ higher than $128 \mathrm{~Hz}$, when $N$ increased, SampEn also increased up to a saturation point. However, this behavior was not observed for $f_{s}=128 \mathrm{~Hz}$ and below. Overall, a first recommendation is that the MAW sampling rate should be equal or higher than $256 \mathrm{~Hz}$ for an appropriate AF organization evaluation with SampEn.

On the other hand, considering the saturation point of $N$, where a constant accuracy was reached for both AF types, a clear relationship between $N$ and $f_{s}$ can be elucidated for all of these situations. As the time period analyzed with SampEn, $T$, is the proportion between $N$ and $f_{s}$, for all these cases we have that $T=\frac{240}{256}=\frac{480}{512}=\frac{960}{1024}=\frac{1920}{2048}=$ $0.9375 \mathrm{~s}$. As a consequence, any combination such that the analyzed time period is longer than one second would be appropriate to evaluate AF organization with SampEn.

With regard to optimal values for $m$ and $r$, note that the region outlined by the combinations with highest accuracy was considerably larger than the one typically recommended in the literature [5]. Nevertheless, these results prove that previous works used adequate values for the SampEn parameters in the estimation of AF organization [3]. However, a better diagnostic precision could be reached for each prediction with a combination of $m$ and $r$ placed within the wider indicated region. An interesting observation is that optimal values reporting the highest accuracy were dissimilar 

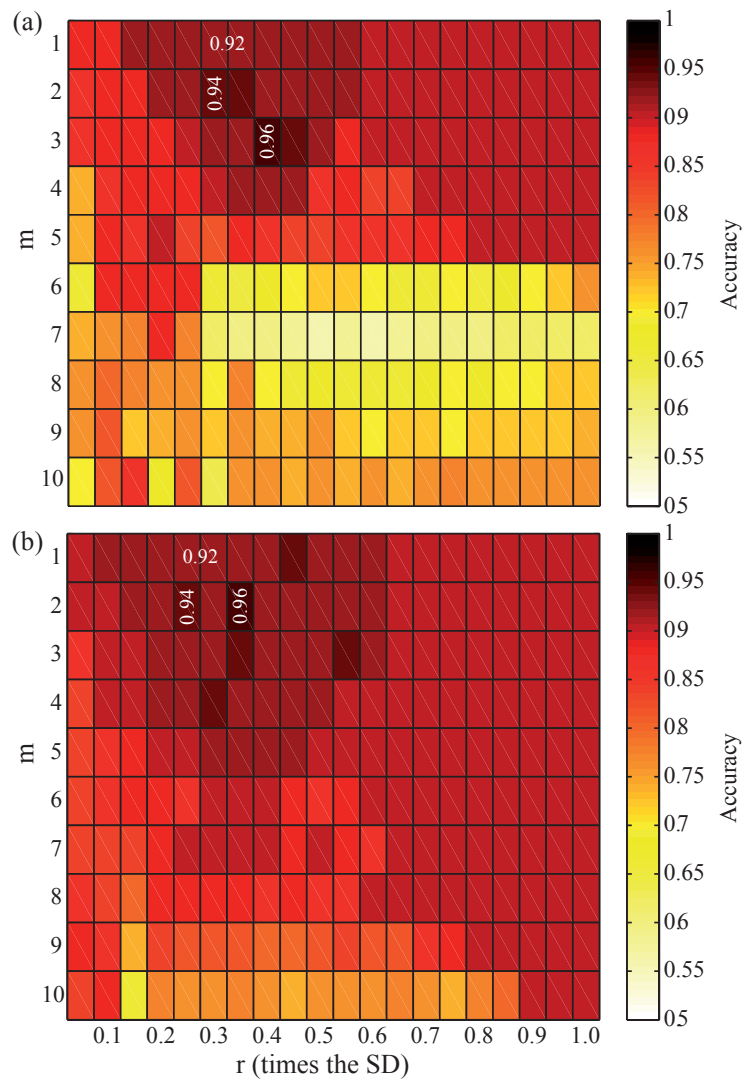

Fig. 2. Diagnostic accuracy color maps for paroxysmal AF termination prediction obtained as a function of $m=1,2, \ldots, 10$ and $r=$ $0.05,0.1,0.15, \ldots, 1$ times the SD of the data, being $N$ and $f_{s}$ (a) 240 samples $-256 \mathrm{~Hz}$ and (b) 960 samples $-1024 \mathrm{~Hz}$, respectively.

depending on the combination of $N$ and $f_{s}$ and on each type of AF. As a consequence, it can be suggested that the optimal combination of $m$ and $r$ has to be searched, within the proposed range, before the first application of SampEn to a non-previously analyzed database. This should only be made with very few recordings of that new database. In this way, the highest predictive ability will be reached.

Finally, both the methodology and the outcomes provided by this study could serve, not only in AF studies, but also as a startup framework in the application of SampEn to other biomedical signal processing scenarios.

\section{ACKNOWLEDGMENTS}

This work was supported by the projects TEC2007-64884, TEC2008-02241 and TEC2010-20633 from the Spanish Ministry of Science and Innovation, PII2C09-0224-5983 and PII1C09-0036-3237 from Junta de Castilla La Mancha, and SAN673/VA02/08 from Junta de Castilla León.

\section{REFERENCES}

[1] S. M. Pincus and A. L. Goldberger, "Physiological time-series analysis: what does regularity quantify?" Am J Physiol, vol. 266, no. $4 \mathrm{Pt}$ 2, pp. H1643-H1656, Apr 1994.

[2] J. S. Richman and J. R. Moorman, "Physiological time-series analysis using approximate entropy and sample entropy." Am J Physiol Heart Circ Physiol, vol. 278, no. 6, pp. H2039-H2049, Jun 2000.
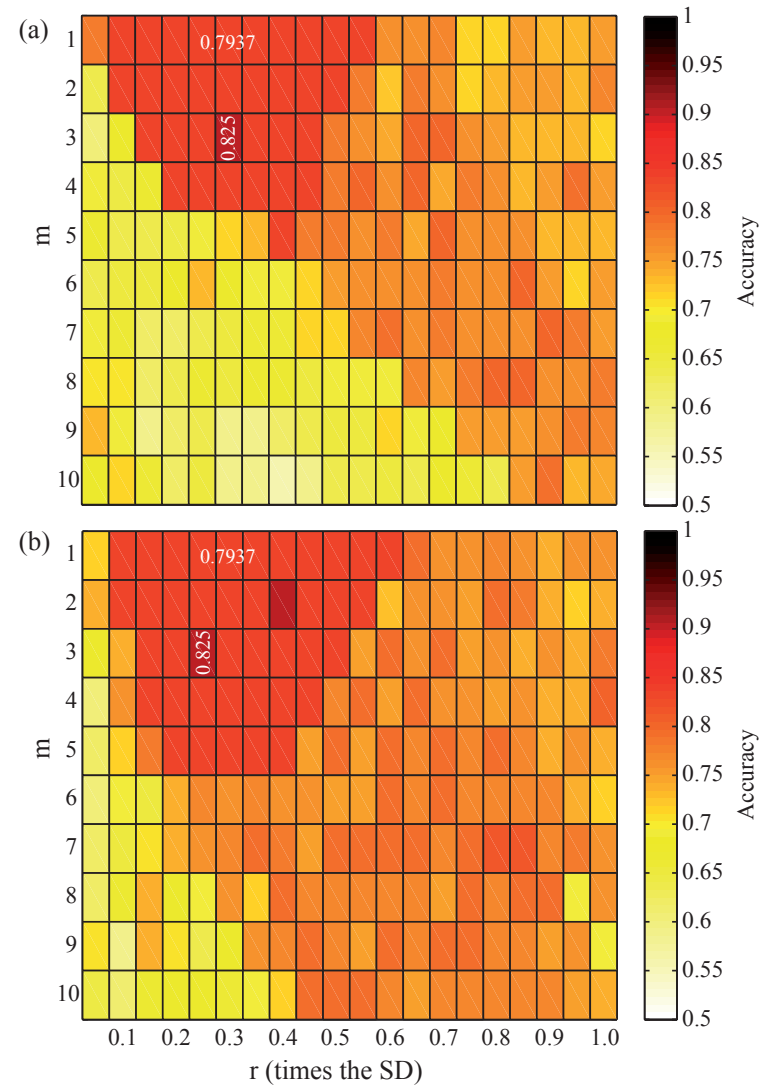

Fig. 3. Diagnostic accuracy color maps for ECV result prediction obtained as a function of $m=1,2, \ldots, 10$ and $r=0.05,0.1,0.15, \ldots, 1$ times the SD of the data, being $N$ and $f_{s}$ (a) 240 samples-256 $\mathrm{Hz}$ and (b) 960 samples- $1024 \mathrm{~Hz}$, respectively.

[3] R. Alcaraz and J. J. Rieta, "A review on sample entropy applications for the non-invasive analysis of atrial fibrillation electrocardiograms," Biomed Signal Process Control, vol. 5, pp. 1-14, 2010.

[4] M. W. Rich, "Epidemiology of atrial fibrillation." J Interv Card Electrophysiol, vol. 25, no. 1, pp. 3-8, 2009.

[5] S. M. Pincus, "Assessing serial irregularity and its implications for health." Ann N Y Acad Sci, vol. 954, pp. 245-267, Dec 2001.

[6] S. M. Pincus and D. L. Keefe, "Quantification of hormone pulsatility via an approximate entropy algorithm." Am J Physiol, vol. 262, no. 5 Pt 1, pp. E741-E754, May 1992.

[7] S. Lu, X. Chen, J. K. Kanters, I. C. Solomon, and K. H. Chon, "Automatic selection of the threshold value $\mathrm{r}$ for approximate entropy." IEEE Trans Biomed Eng, vol. 55, no. 8, pp. 1966-1972, 2008.

[8] A. Bollmann, D. Husser, L. Mainardi, F. Lombardi, P. Langley, A. Murray, J. J. Rieta, J. Millet, S. B. Olsson, M. Stridh, and L. Sörnmo, "Analysis of surface electrocardiograms in atrial fibrillation: Techniques, research, and clinical applications." Europace, vol. 8, no. 11, pp. 911-926, Nov 2006.

[9] A. L. Goldberger, L. A. Amaral, L. Glass, J. M. Hausdorff, P. C. Ivanov, R. G. Mark, J. E. Mietus, G. B. Moody, C. K. Peng, and H. E. Stanley, "Physiobank, physiotoolkit, and physionet: Components of a new research resource for complex physiologic signals." Circulation, vol. 101, no. 23, pp. E215-E220, Jun 2000.

[10] S. M. Pincus, "Approximate entropy as a measure of system complexity." Proc Natl Acad Sci USA, vol. 88, no. 6, pp. 2297-2301, 1991.

[11] D. E. Lake, J. S. Richman, M. P. Griffin, and J. R. Moorman, "Sample entropy analysis of neonatal heart rate variability." Am J Physiol Regul Integr Comp Physiol, vol. 283, no. 3, pp. R789-R797, Sep 2002.

[12] M. J. Lewis and A. L. Short, "Sample entropy of electrocardiographic RR and QT time-series data during rest and exercise." Physiol Meas, vol. 28, no. 6, pp. 731-744, Jun 2007. 